\title{
EQUATING $k$ MAXIMUM DEGREES IN GRAPHS WITHOUT SHORT CYCLES
}

\author{
Maximilian Fürst, Michael Gentner, \\ Simon JäGer, Dieter Rautenbach \\ Institute of Optimization and Operations Research \\ Ulm University, Germany \\ e-mail: maximilian.fuerst@uni-ulm.de \\ michael.gentner@uni-ulm.de \\ simon.jaeger@uni-ulm.de \\ dieter.rautenbach@uni-ulm.de
}

AND

Michael A. Henning

Department of Pure and Applied Mathematics University of Johannesburg, South Africa

e-mail: mahenning@uj.ac.za

\begin{abstract}
For an integer $k$ at least 2, and a graph $G$, let $f_{k}(G)$ be the minimum cardinality of a set $X$ of vertices of $G$ such that $G-X$ has either $k$ vertices of maximum degree or order less than $k$. Caro and Yuster [Discrete Mathematics 310 (2010) 742-747] conjectured that, for every $k$, there is a constant $c_{k}$ such that $f_{k}(G) \leq c_{k} \sqrt{n(G)}$ for every graph $G$. Verifying a conjecture of Caro, Lauri, and Zarb [arXiv:1704.08472v1], we show the best possible result that, if $t$ is a positive integer, and $F$ is a forest of order at most $\frac{1}{6}\left(t^{3}+6 t^{2}+17 t+12\right)$, then $f_{2}(F) \leq t$. We study $f_{3}(F)$ for forests $F$ in more detail obtaining similar almost tight results, and we establish upper bounds on $f_{k}(G)$ for graphs $G$ of girth at least 5 . For graphs $G$ of girth more than $2 p$, for $p$ at least 3 , our results imply $f_{k}(G)=O\left(n(G)^{\frac{p+1}{3 p}}\right)$. Finally, we show that, for every fixed $k$, and every given forest $F$, the value of $f_{k}(F)$ can be determined in polynomial time.
\end{abstract}

Keywords: maximum degree, repeated degrees, repetition number.

2010 Mathematics Subject Classification: 05C05, $05 \mathrm{C} 07$. 


\section{REFERENCES}

[1] M.O. Albertson and D.L. Boutin, Lower bounds for constant degree independent sets, Discrete Math. 127 (1994) 15-21. doi:10.1016/0012-365X(92)00463-2

[2] B. Bollobás and A.D. Scott, Independent sets and repeated degrees, Discrete Math. 170 (1997) 41-49. doi:10.1016/0012-365X(95)00355-Z

[3] Y. Caro, J. Lauri and C. Zarb, Equating two maximum degrees, manuscript. arXiv:1704.08472v1

[4] Y. Caro, A. Shapira and R. Yuster, Forcing k-repetitions in degree sequences, Electron. J. Combin. 21 (2014) \#P24.

[5] Y. Caro and R. Yuster, Large induced subgraphs with equated maximum degree, Discrete Math. 310 (2010) 742-747.

doi:10.1016/j.disc.2009.09.003

[6] Y. Caro and D.B. West, Repetition number of graphs, Electron. J. Combin. 16 (2009) \#R7.

[7] M. Miller and J. Širán̆, Moore graphs and beyond: A survey of the degree/diameter problem, Electron. J. Combin. 20 (2013) \#DS14v2.

Received 29 November 2017

Revised 15 May 2018

Accepted 15 May 2018 